\title{
Improved Representation of Destructive Spacecraft Re-entry from Analysis of High Enthalpy Wind Tunnel Tests of Spacecraft and Equipment
}

\author{
James C. Beck ${ }^{a *}$, Ian Holbrough ${ }^{\mathrm{a}}$, Thorn Schleutker ${ }^{\mathrm{b}}$, Ali Guelhan ${ }^{\mathrm{b}}$ \\ a Belstead Research Limited, 387 Sandyhurst Lane, Ashford, TN25 4PF, UK, james.beck@,belstead.com, \\ ian.holbrough@belstead.com \\ b Supersonic and Hypersonic Technology, Deutsches Zentrum fuer Luft- und Raumfart, Linder Hoehe, 51147 Koeln, \\ Germany, thorn.schleutker@dlr.de, ali.guelhan@dlr.de \\ * Corresponding Author
}

\begin{abstract}
The assessment of casualty risk in destructive re-entry has historically been performed purely by simulation using heating correlations, which only have verification on basic shapes, and estimated phenomenology for fragmentation. As the application of space debris mitigation requirements is expected to result in a higher number of re-entries, this has brought a stricter enforcement of the casualty risk guidelines to proposed missions. In turn, this has increased the interest in designing spacecraft to demise in re-entry in order to allow uncontrolled re-entries to be performed. Initial testing of spacecraft materials and basic structures has demonstrated that the destructive re-entry tools do not capture the correct physics to be able to assist design in a meaningful way, and therefore, some means to improve the representativeness of the tools is required. The current understanding of the phenomenology of the fragmentation and demise processes is limited. As a consequence, it is vital to perform appropriate tests in order to improve the capability of the tools to assist the design process. To this end, a set of destructive tests on spacecraft materials, structures and components has been performed in an arc-heated supersonic wind tunnel. These tests include the first destructive wind tunnel tests ever performed on a complete nano-satellite and a reaction wheel. From these tests, it has been determined that the failure of aluminium structures is highly dependent upon the behaviour of the protective metal oxide layer, and that this can be catastrophic in nature. The tests on the nano-satellite have shown that the structure can be supported by stainless steel spacers between the electronics cards, and that glass fibre reinforced plastic PCBs are more resistant to melting than had been anticipated. The reaction wheel test has shown that the connections between parts are critical to the fragmentation and demise processes, as the glued housing separates quickly, well before melt temperature is reached at the joint. It has also demonstrated the importance of radiative cooling, as the flywheel and ball-bearing unit have survived a test at over $800 \mathrm{~kW} / \mathrm{m}^{2}$ with little damage.

Keywords: aerothermodynamics, aerothermal testing, design for demise, fragmentation, re-entry, spacecraft equipment

\section{Introduction}

Concern over the increase of space debris has led to a set of guidelines requiring the removal of satellites and upper stages from Low Earth Orbit (LEO) at the end of their useful life. For the majority of spacecraft, this removal will involve a de-orbit and destructive reentry into the Earth's atmosphere.

It is well known that some spacecraft parts have survived the re-entry process, with components from Skylab [1] as well as a number of upper stage propellant tanks having been recovered from landing sites [2]. This being the case, the guidelines allow a casualty risk of 1:10000 for an uncontrolled re-entry to be permitted. With the additional cost of a controlled re-entry being potentially significant, especially if a larger launcher is then required, effort is now being placed into the design of spacecraft to increase their demisability during reentry.

This field of design-for-demise is relatively new, and only two testing campaigns [3] were carried out prior to 2013. These focussed on specific material tests

for particular spacecraft. With the greater interest in design-for-demise, there has been a move towards increased testing. The resulting output is being used to validate and improve the current generation of models which support the assessment of the casualty risk from re-entry. The test campaigns performed so far have mainly focussed on the behaviour of basic spacecraft materials in isolation, with only very basic tests having been performed to date on sandwich structures and joints. It is worth noting that there is a large knowledge gap in the phenomenology of fragmentation and demise, and the accuracy of casualty risk prediction by the current generation of simulation tools is not known.

The more recent experimental demisability testing work started with two parallel ESA studies on the characterisation of a number of common spacecraft materials. Interestingly, these two test campaigns took very different approaches. Both teams assessed five standard materials (aluminium, stainless steel, titanium, silicon carbide and CFRP), as well as selected other, more novel, materials of interest. The CHARDEM
\end{abstract}


project [4] obtained high quality temperature dependent material properties, and performed their wind tunnel demise tests using relatively thick monolithic samples at relatively high heat fluxes in order to assess the coarse demise phenomena. The heat fluxes were sufficiently high to ensure that demise was observed for all materials except silicon carbide. The CoDM project, however, focussed on lower tumble-average heat fluxes on thin samples within a sample holder such that an isothermal, or one-dimensional, heat distribution could be obtained through the material [5]. The low fluxes used allowed a number of steady state conditions to be obtained permitting an assessment of the material catalycity, which can potentially reduce the heat flux level to the material. Emissivity measurements of the samples were also performed, and these clearly demonstrated that oxidised, post-test samples have higher emissivities than those which are generally applied in destructive re-entry codes. The emissivity and catalycity data collected demonstrated that the current codes were overly optimistic in their prediction of material demise.

In general, both activities found that the nature of the material demise is not well understood, even for simple monolithic metallic materials, and that the model representations of more complex materials such as CFRP and glass-laminates is quite poor. Indeed, it is not clear that the demise processes themselves are fully understood, and thus it cannot be claimed that these processes are modelled correctly. Of the materials tested, the priority is to provide improved data on CFRP materials, especially in the behaviour of the material once it has fully charred. It is currently expected to break up under aerodynamic forces, and is modelled as such, but this has not been confirmed experimentally.

A very different study was carried out under the ESA CleanSat building blocks activities on basic phenomenology of joints and spacecraft fragmentation [6]. This study focussed on the behaviour of sandwich materials, specifically the joints employing adhesive materials which are expected to have significantly reduced strength at high temperature. The forces required to remove the facesheets from the honeycomb core at high temperature were found to be reasonably small, at a few Newtons at temperatures of $200^{\circ} \mathrm{C}$. Thus, peeling of the facesheets under the forces caused by the inertia of the relatively heavy equipment attached to panels is a distinct possibility during re-entry. This suggests that relatively small forces could cause separation of the spacecraft equipment, such that large items would not be expected to remain connected to the platform.

More recently, an ESA study assessing the high altitude fragmentation of spacecraft [7] has confirmed these findings, and has shown that the failures of potted inserts can occur early in a re-entry from a decaying circular orbit.

This testing work has served to demonstrate that the current understanding of destructive re-entry processes is relatively basic, and that improved knowledge of the material response, failure and fragmentation processes are required in order to provide good support to spacecraft designers and regulators in assessing the performance of design-for-demise efforts. To this end, the test campaign performed here has three main aspects; improved representation of common materials, improved modelling of spacecraft structures and a first understanding of the demise behaviour of real spacecraft components.

\section{Test Planning and Setup}

\subsection{L2K Facility}

The L2K facility is one of the wind tunnels in the Hypersonic Technology Department of DLR Cologne. Along with the higher pressure L3K facility, it is part of the LBK facility, as shown in Fig.1 [8]. Significant advantages of the LBK tunnels are that they are supersonic and run at Mach numbers of between 3 and 8. While this is well below the flight Mach number ( Mach 25 ), it is more representative than many archeated facilities, which run in either the subsonic or transonic range. This allows something of the shock structure to be understood.

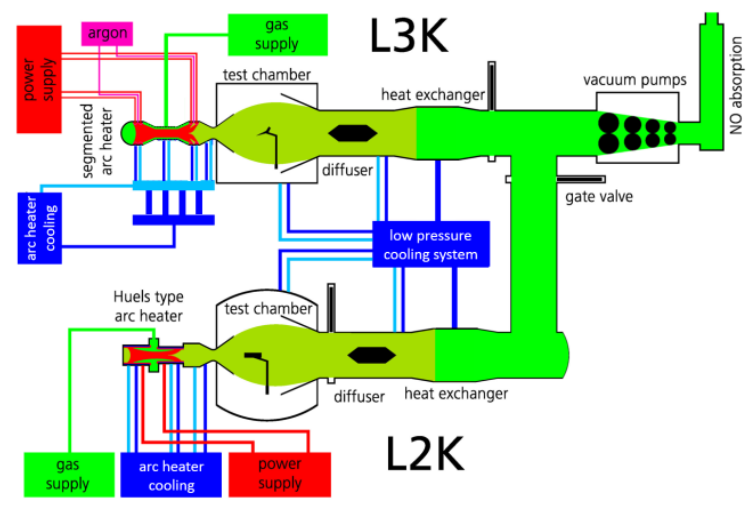

Fig. 1. LBK Facilities.

The $\mathrm{L} 2 \mathrm{~K}$ test leg is equipped with a Huels type arc heater with a maximum electrical power of $1.4 \mathrm{MW}$ which is used to transfer the working gas to a high enthalpy state. At its downstream end the arc heater is connected to a convergent-divergent nozzle which accelerates the gas to hypersonic velocities. After passing the nozzle exit the accelerated gas enters the test chamber where it forms a free jet. Models can be exposed to cold wall heat flux rates of up to $3 \mathrm{MW} / \mathrm{m}^{2}$ at stagnation pressures of up to $16 \mathrm{kPa}$. 
As well as the standard stagnation point configuration, a shear test setup is also available in the LBK tunnels. In this configuration, the heat fluxes are lower, and vary with the running length on the 'wedge' sample holder, but this setup is more accessible for the assessment of joints and sandwich-type material failures.

\subsection{Test Selection}

The two materials which will be investigated are aluminium, as the major structural component of spacecraft, and CFRP which is becoming more prevalent as a structural material. A large fraction of the overall spacecraft mass, often more than half, can be expected to consist of these two materials.

Sandwich panels are the basis of the structure of the majority of spacecraft. As such, the failure of the sandwich panels, and the resultant opening of the spacecraft is critical to the release of the internal components, and thus their ensuing demise. It is worth noting that there is currently no specific model for the failure and demise of sandwich materials, with either aluminium or CFRP facesheets, in any destructive reentry code.

Although some testing on the demise phenomenology of joints has been performed, no basic testing has been performed on a complete spacecraft component. It is important to understand the differences in the demise behaviour which derive from the compound nature of the components, and the presence of different materials. The components selected for study are a set of electronics cards in a $1 \mathrm{U}$ CubeSat and a reaction wheel, which has been identified as a critical component in previous studies [9].

\subsection{Test Conditions}

The key aspects in the demise of a spacecraft are the spacecraft fragmentation, which occurs at altitudes of approximately $80 \mathrm{~km}$, and the subsequent demise of the separated components which generally occurs between $60 \mathrm{~km}$ and $80 \mathrm{~km}$ altitude. Both of these aspects are of interest for this campaign, as some of the testing is on aspects intended to improve fragmentation, and others on aspects intended to improve the subsequent component demise. Therefore, more than one test condition is required.

The conditions experienced by the intact spacecraft during re-entry are driven by the ballistic coefficient of the vehicle, and the heating rates to joints are dependent upon the tumbling motion. However, due to the low atmospheric density, the variation in the conditions at fragmentation is relatively low. Therefore, a generic fragmentation condition can be reasonably well defined. This is not the case, however, for the released components. For these items, the heating is highly dependent upon the altitude at which the component is released from the spacecraft, the ballistic coefficient of the component and the component size. Therefore, no single condition can be expected to capture relevant demise conditions for all components.

Derivation of the spacecraft environmental conditions at the expected fragmentation altitudes has been performed as part of [7]. Additionally, a comprehensive study of component conditions and demise has been carried out as part of [5], spanning the space of the expected conditions, inclusive of stagnation point and tumble-averaged heating levels. From these works, two conditions can be defined, as the aerodynamic behaviour of the component is different from the static nature of the wind tunnel test. The relevant conditions are given in Table 1 and the selected test conditions in Table 2.

\begin{tabular}{lcll} 
Table 1. Relevant Environmental Conditions. \\
\hline & Fragmentation & $\begin{array}{l}\text { Demise } \\
\text { (stagnation) }\end{array}$ & $\begin{array}{l}\text { Demise } \\
\text { (tumble- } \\
\text { average) }\end{array}$ \\
\hline $\begin{array}{l}\text { Heat Flux } \\
\left(\mathrm{kW} / \mathrm{m}^{2}\right)\end{array}$ & $20-100$ & $400-2000$ & $100-500$ \\
$\begin{array}{l}\text { Stagnation } \\
\text { Pressure } \\
(\mathrm{Pa})\end{array}$ & $1-1000$ & $1000-$ & $1000-$ \\
\hline
\end{tabular}

Table 2. Selected Test Conditions

\begin{tabular}{lllll}
\hline & TC1 & TC2 & TC3 & Shear \\
\hline Heat Flux $\left(\mathrm{kW} / \mathrm{m}^{2}\right)$ & 100 & 200 & 800 & 100 \\
$\begin{array}{l}\text { Stagnation Pressure } \\
(\mathrm{Pa})\end{array}$ & 1000 & 1000 & 5000 & 4000 \\
\hline
\end{tabular}

\subsection{Test Samples}

The test samples were designed specifically for this campaign. Previous testing on aluminium 7075 samples considered either thin coupons or thick monolithic samples, neither of which are representative of spacecraft structures. To address this problem, a $100 \mathrm{~mm}$ diameter top-hat model with an $80 \mathrm{~mm}$ top and $2 \mathrm{~mm}$ thickness has been used, which is shown in Fig. 2.

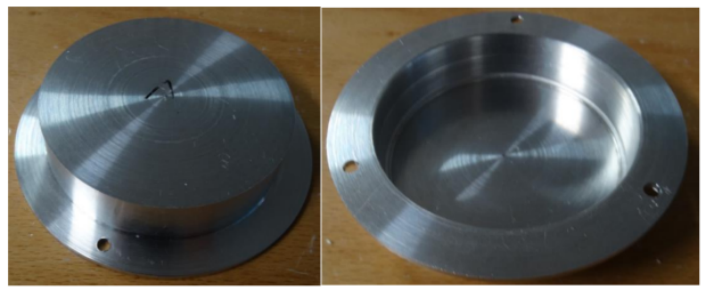

Fig. 2. Top-hat aluminium sample.

For testing it is important to use flat sheets of CFRP to circumvent the issue that the carbon fibres are strong in tension. If other geometries were used the hoop 
stresses in circular CFRP pieces will not be representative of the behaviour of the flat panels, which are usually seen on spacecraft. Therefore, for this activity it has been decided to focus on sandwich panel facesheets, which are the major use of CFRP on spacecraft. An example of the $100 \times 100 \mathrm{~mm}$ sample is shown in Fig. 3.

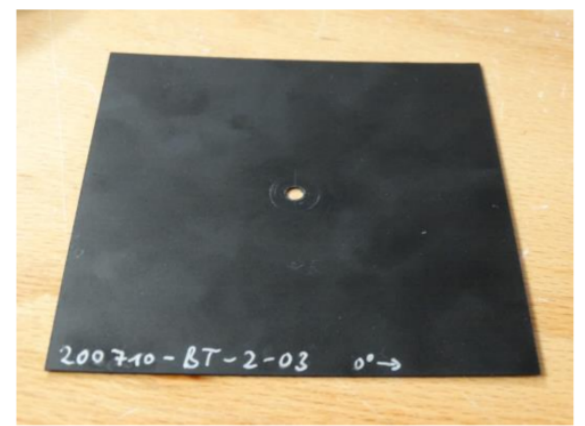

Fig. 3. CFRP Facesheet Sample.

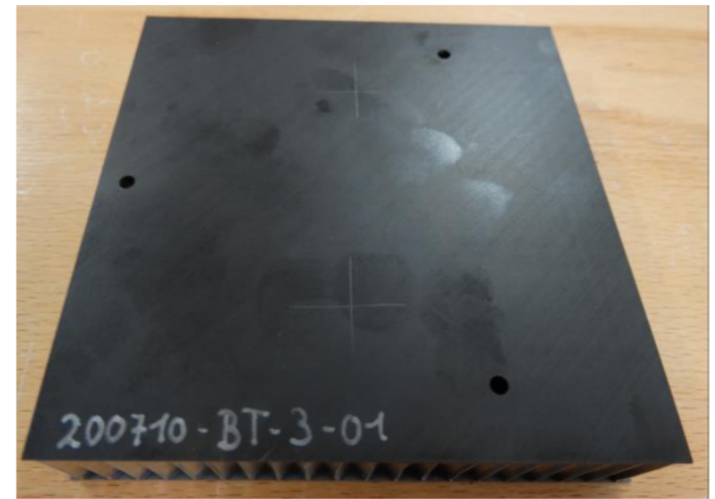

Fig. 4. CFRP Sandwich Panel.

Dialogue with another ESA project [7] has suggested use of sandwich panel samples of $20 \mathrm{~mm}$ thickness as this is the standard for spacecraft external panels. It is worth noting that this results in panels that are relatively thick in relation to their $100 \mathrm{~mm}$ dimension and raises the question as to whether the influence of the panel edges is too large. However, thinner sandwich panels are not standard, and it was decided to use standard panels for this test campaign given that the knowledge of panel demise behaviour is still very poor. A sample is shown in Fig. 4. The honeycomb material selected is a standard $3 / 16$ inch $(4.8 \mathrm{~mm})$ cell size with a $25 \mu \mathrm{m}$ foil thickness and is manufactured from AL5056 alloy. A Hexcel Redux $312 \mathrm{UL}$ film adhesive is used to join the facesheet to the honeycomb. The CFRP facesheet and honeycomb samples have been obtained from INVENT. The sandwich panel tests reported here use 4 ply $(0.3 \mathrm{~mm}$ thick) facesheets, whereas the bare facesheets are 8 ply $(0.6 \mathrm{~mm}$ thick).
With a reaction wheel having been identified as a key priority for testing, significant effort was placed into obtaining a suitable model. In dialogue with engineers from Rockwell Collins, a test object based on an RSI1.6 reaction wheel with a diameter of $120 \mathrm{~mm}$ was proposed for testing. This has a smaller flywheel than the spacecraft flight reaction wheel, but has a similar architecture and utilises a similar ball bearing unit and the same flywheel material. Therefore, representative tests are expected. The test object is shown in Fig. 5.

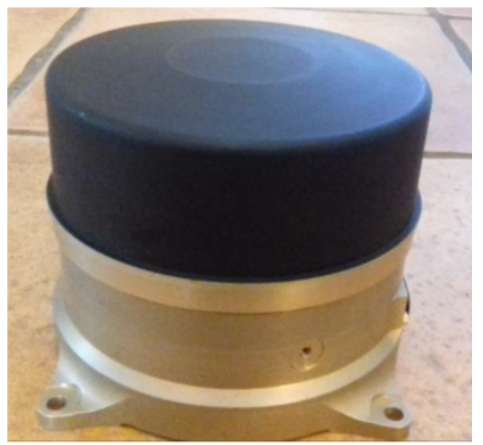

Fig. 5. Reaction Wheel Engineering Model.

A CubeSat test object has been constructed by purchasing a structure from EnduroSat and attaching a number of electronics cards, side panels and a dummy solar array. The base structure is aluminium and the four electronics cards used (a sound card, logic board, power supply and mobile phone battery) have been attached using the steel spacer rods as shown in Fig. 6.
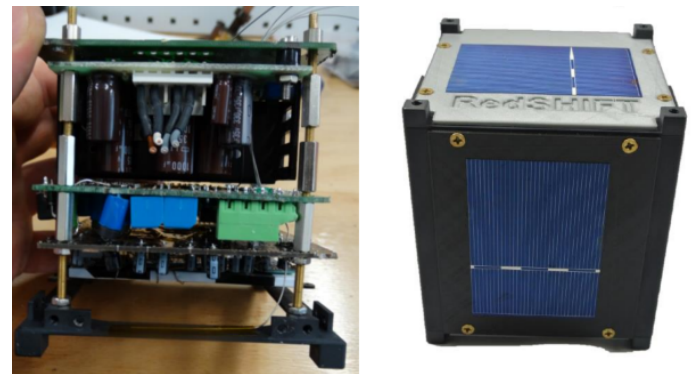

Fig. 6. CubeSat Test Object.

The samples are instrumented using type $\mathrm{K}$ thermocouples, and the tests are observed with highdefinition video, an infra-red camera and a high-speed video camera.

\section{Test Results}

A subset of the full campaign of 22 tests are reported here, as summarised in Table 3 . Stagnation point and shear tests have been performed on aluminium and CFRP samples, CFRP sandwich samples have been tested at an angle of attack and as well as unique tests 
on a reaction wheel engineering model and full CubeSat.

Table 3. Test Matrix.

\begin{tabular}{lll}
\hline Sample & Condition & Configuration \\
\hline Aluminium Top Hat & TC1 & Stagnation \\
Aluminium Plate & Shear & $15^{0}$ Attack \\
CFRP Facesheet & TC1 & Stagnation \\
CFRP Facesheet & Shear & $15^{0}$ Attack \\
CFRP Sandwich & TC1 & $30^{\circ}, 60^{\circ}, 90^{\circ}$ \\
Reaction Wheel & TC1, TC2 & Stagnation \\
Reaction Wheel Parts & TC3 & Stagnation \\
CubeSat & TC1, TC2 & Stagnation \\
\hline
\end{tabular}

\subsection{Aluminium Material Tests}

During the heat up of the aluminium top hat, a number of interesting observations were made. Fig. 7 shows a red glow around the sample, which is understood to be due to the outgassing of the zinc components in the AL7075 alloy. The thermocouple data demonstrates that the top hat is close to isothermal and that the temperature at the start of the outgassing is $330^{\circ} \mathrm{C}$ and that the outgassing is completed at about the time that the melt temperature of aluminium is reached. Thermocouple TC1 is on the front face with TC2 and TC3 on the flange.

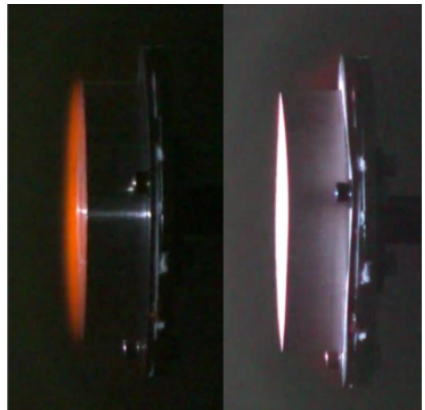

Fig. 7. Top Hat During (left) and after Outgassing.

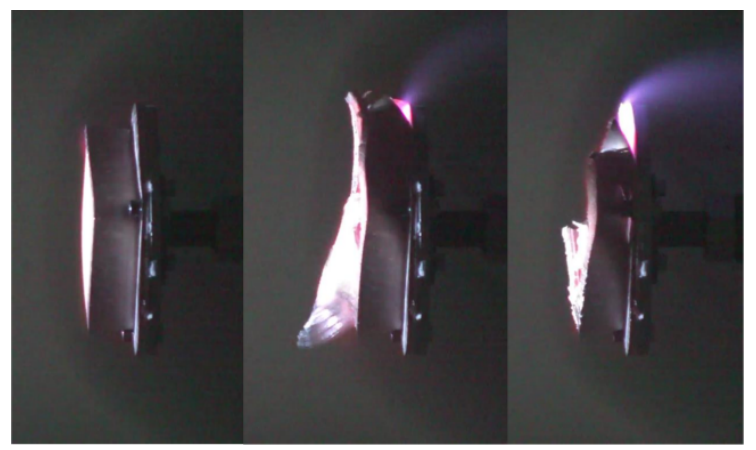

Fig. 8. Failure of Aluminium Top Hat.

The top hat is then observed to undergo a catastrophic failure. As shown in Fig. 8, the top of the hat moves inwards and the bottom outwards. The top hat is blown apart, the walls fail and only a few remnants attached by the screws to the sample holder remain. No large parts were retrieved from the wind tunnel, confirming that the failure was indeed catastrophic.

This suggests that contiguous aluminium parts may not melt resulting in shear of small droplets, but can fail in some form of more catastrophic manner which is essentially caused by the tearing of the protective oxide layer under deformation of the molten material it contains.

Performing a simple isothermal model rebuild on the top hat test using the SAM code [10] and the heat fluxes calculated via CFD rebuilds yields Fig. 9. Using the nominal $100 \mathrm{~kW} / \mathrm{m}^{2}$ flux provides a very good reconstruction of the front face temperature profile. It is interesting to note that the point at which the code would predict a full demise of the top hat is about $40 \mathrm{~s}$ after the catastrophic failure seen. This suggests that the aluminium is some way from being fully molten when it fails catastrophically, but that it occurs after the melting point is reached at the surface, due to the protective oxide layer. In this case the catastrophic failure occurs once approximately one-third of the material is predicted to have melted, which suggests that current models of aluminium failure in re-entry tools could be conservative.

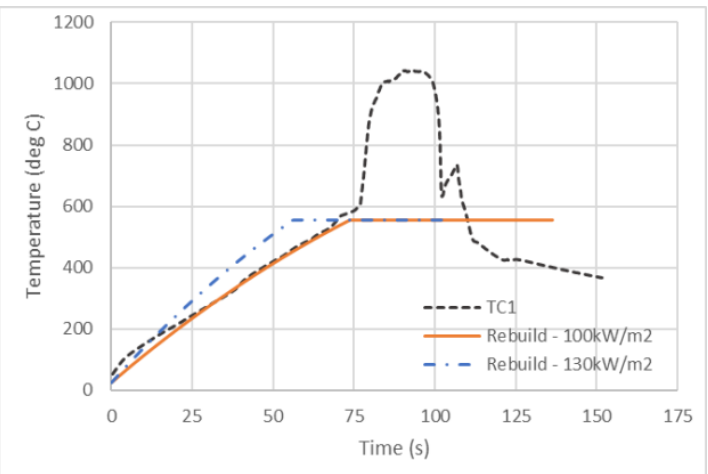

Fig. 9. Aluminium Top Hat Test Rebuild.

The strength of the aluminium oxide layer is confirmed in the shear tests. In this case outgassing is evident in the production of small bubbles which gradually grow as shown in Fig. 10. The motion of the bubbles downstream and their bursting can be seen clearly, and therefore, it is likely that these are gas bubbles. These should be distinguished from the larger bulges of liquid aluminium which can be seen in the right hand image of Fig. 10.

The shear forces are estimated at $25 \mathrm{~N} / \mathrm{m}^{2}$ via CFD rebuilds using the TINA code, and it is clear that these forces are insufficient to tear the oxide layer. Indeed, the molten aluminium inside the oxide layer is observed to sag under gravity, which eventually causes the failure of 
the oxide 'bag' and the release of the molten aluminium contained within.

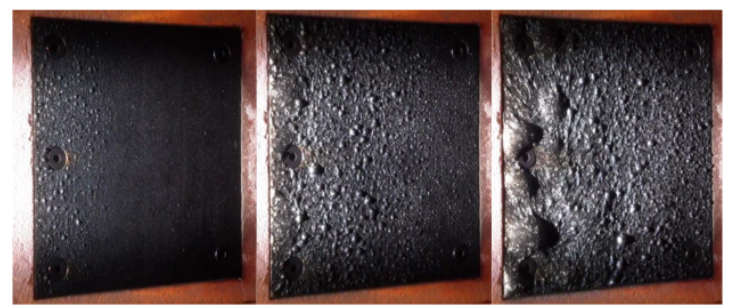

Fig. 10. Bubbles in Aluminium Shear Test.

In this test, there are some useful images from the high speed camera. It is evident that the oxide bag does not simply tear, but continually tears and reforms, with the mass being released in bursts.

The rebuilds of the shear test, again using a simple isothermal model in SAM, suggest that at the time of the material slump under gravity approximately $40 \%$ of the aluminium mass is molten, which is reasonably consistent with the timing of the oxide failure in the top hat test. This suggests that the motion of the molten aluminium drives the oxide layer failure, and the subsequent demise of the aluminium structure.

\subsection{CFRP Material Tests}

The purpose of the stagnation configuration CFRP test was to assess the likelihood of a charred CFRP material, where the matrix is essentially completely ablated, losing its structural integrity under aerodynamic load. The idea was that the carbon fibres may break, resulting in fragments reaching the ground that pose no risk. In practice, the test did not proceed as expected. As shown in Fig. 11, the panel essentially folded in a 2D manner, and very little flutter of the carbon fibre was observed as the facesheet retained something of its structural integrity. This continued until the bending of the panel was significant enough that sufficient fibres could be removed leading the panel to break.

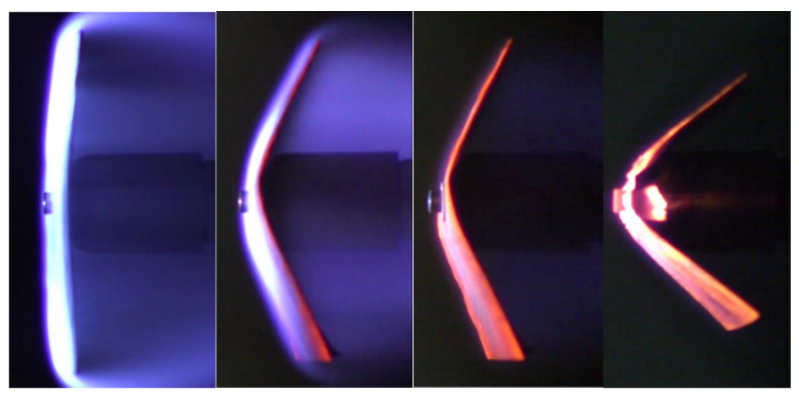

Fig. 11. Sequence of Stills from CFRP Test.

As soon as the facesheet was placed into the flow, the outgassing due to pyrolysis started. The plies on the facesheet could be seen to be slightly lifted such that the gas could escape, resulting in a rippled surface and some delamination at the corners. Within 10 s of the flow start, the sheet had bent approximately 20 degrees to the vertical in a single preferred direction.

It was evident that the heat fluxes are higher at the edges than at the centre of the sample, which is consistent with the CFD calculations and the thinning of the boundary layer with the acceleration around the corner. With the bending of the sample, it could also be observed that the heating to the stagnation region was higher than the heating on the flat, inclined surface.

The first major fibre removal occurred after more than 10 minutes of test time, showing that the CFRP material is highly resistant to the heat flux. It was noticeable that the increased bending tightened the radius of the stagnation region and resulted in increased heat fluxes and more fibre removal.

In the shear test, the pyrolysis of the CFRP matrix again begins as soon as the sample enters into the plasma flow, with outgassing immediately evident. The surface of the facesheet becomes uneven due to the plies being lifted by the pyrolysis gas pressure as it finds routes through to the surface to escape. The outgassing and the uneven surface are shown clearly in Fig. 12.
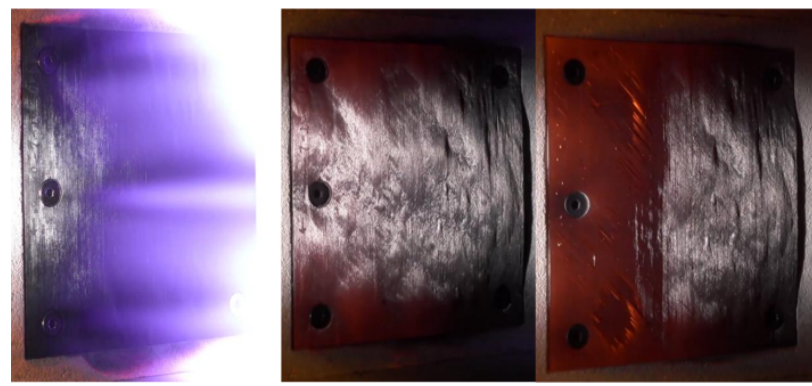

Fig. 12. Stills from CFRP Shear Test.

Once the outgassing is complete, the fibres can be seen to be removed layer-by-layer from the front of the sample. Whereas $25 \mathrm{~N} / \mathrm{m}^{2}$ was insufficient shear to damage the oxide layer on the aluminium samples, it is sufficient to augment the loss of oxidising carbon fibres from facesheets. This mechanical effect can be observed by the different plies having fibres at different orientations, at least three such layers are visible in the right hand pane of Fig. 12. The temperature of the sample can also be seen to increase as it thins.

The removal of the material from the bolts is also interesting as there is no tearing involved. Previous work with aluminium facesheets has shown that the facesheet needs to tear to be removed from an insert, where a CFRP facesheet does not [6]. This is consistent with the observations here, and the most likely explanation is the difference in the coefficient of thermal expansion between the CFRP material and the steel bolts. 
The temperature measurements show a steady state of $800^{\circ} \mathrm{C}$, which would result in the CFRP being assumed to be fully charred in a few tens of seconds within the current model in the SAM code. At this point it is assumed that the panel is relatively weak and would no longer pose a casualty risk were it to reach the ground. Given that these facesheets are very light, this is indeed likely to be the case. However, it is clear that the integrity of the facesheets is maintained well after the pyrolysis gassing is complete. Therefore, these items can be expected to remain essentially intact through the re-entry, especially as they will rapidly slow due to their low ballistic coefficient. As a consequence, the current model is considered over-optimistic in its consideration of the demise of the facesheet. However, as shown in Fig. 13, the heat up of the sample and the radiative equilibrium temperature in the early stages of the test are well represented, even using a simple 1D solution. The key open point for casualty risk assessment is the criterion by which the composite sample can be considered to be demised.

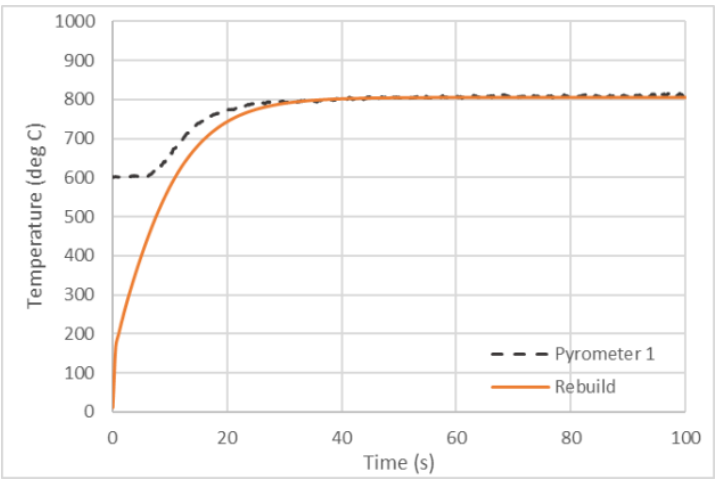

Fig. 13. CFRP Stagnation Test Rebuild.

\subsection{Sandwich Panel Tests}

The first of the CFRP sandwich panel tests is performed in the standard stagnation configuration with the panel normal to the flowfield. Outgassing begins $2 \mathrm{~s}$ after the sample is inserted into the flow and stops at $11 \mathrm{~s}$ at which point the second ply of carbon fibres is visible. This can be seen in the first two panes of Fig. 14. The final pane shows the compression of the honeycomb which begins at $26 \mathrm{~s}$ and is complete at $46 \mathrm{~s}$. Melting of the honeycomb at the top edge of the sample can also be observed.

Some of the compressed honeycomb remained within the facesheets and was retrieved after the end of the test. This demonstrates the relatively low mass of aluminium which is used in the honeycomb core of these standard panels. Also worth noting is that the hexagonal pattern was visible in the glue, which shows that the glue has not been fully oxidised, and that only slight damage to the facesheet was observed.

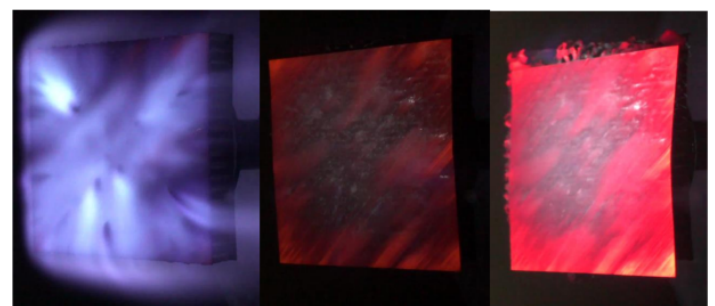

Fig. 14. Stills from CFRP Sandwich Test.

Running the CFRP sandwich panel at a $60^{\circ}$ angle of attack was expected to allow sufficient flow between the facesheets that the honeycomb would recede and the upper facesheet would be removed from the panel. However, whilst the honeycomb was seen to recede within the panel as the flow penetrated, the front facesheet collapsed onto the back facesheet, as there was insufficient lateral force to remove it at this angle of attack.

Moving to a $30^{\circ}$ angle of attack resulted in removal of the facesheet as demonstrated in Fig. 15. Of particular interest here is the recession of the honeycomb. Previous testing of panels with inserts [7] has suggested that the honeycomb removal is dominated by edge effects, and that the flow into the panel between the facesheets is minimal. This test suggests that this observation is driven by the presence of the insert/potting material, as it is not seen here. In this test, the recession can be well described by an elliptical profile, which is consistent with a laminar heat flux profile. This suggests clearly that the recession of the honeycomb is driven by the incoming heat fluxes and that the edge effects are not a factor.

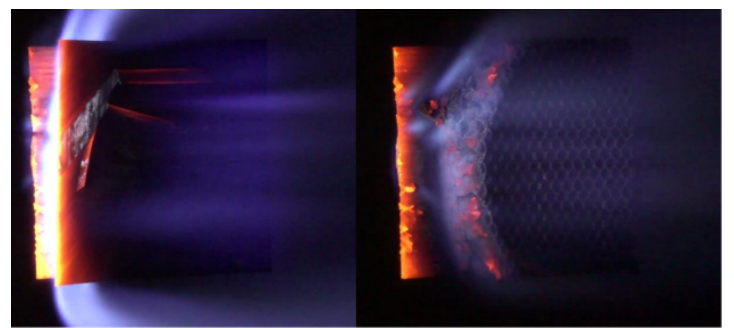

Fig 15. Facesheet Removal in Panel Test at $30^{\circ}$.

The rebuilds here have been performed using a 1D conduction/ablation model (CAM) which has been embedded within the SAM simulation framework using the area averaged flux value of $120 \mathrm{~kW} / \mathrm{m}^{2}$ derived from CFD runs. The 1D model consists of two facesheets of $0.3 \mathrm{~mm}$ thickness and a proxy aluminium core of $19.4 \mathrm{~mm}$ thickness which uses a density of $40 \mathrm{~kg} / \mathrm{m}^{3}$ and a thermal conductivity of $1.2 \mathrm{~W} / \mathrm{mK}$ (the remaining properties used are those of standard aluminium). The layup of the model is given in Fig. 16, with the facesheet thicknesses varied to be appropriate to the specific test. 


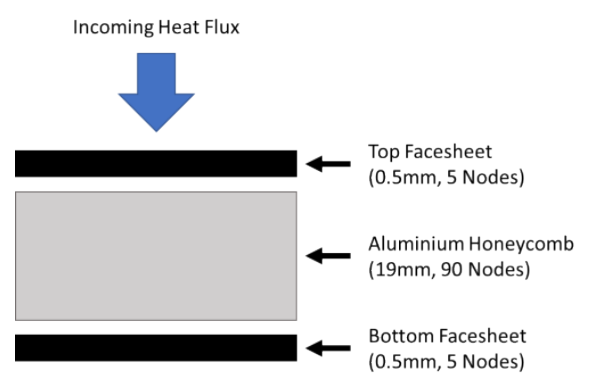

Fig. 16. 1D Sandwich Panel Model Layup.

Fig. 17 shows that the front face temperatures are very well captured in the test with the panel at $90^{\circ}$ to the flow. The back face temperature is also extremely well represented in the model, suggesting that the conductivity of the honeycomb and the density of the proxy 1D material provides a very accurate thermal diffusivity. It is interesting to note that there are no clear jumps in the thermocouple reading from the honeycomb collapse, which might be expected. Instead, the thermal link between the front and back faces is consistent even during the honeycomb collapse, which is not explicitly modelled. Such an excellent rebuild is not generally expected with the simplified models used.

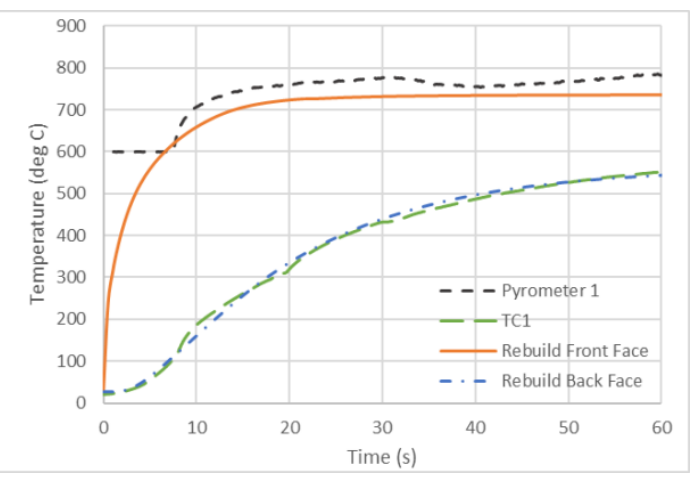

Fig. 17. Rebuild of CFRP Panel Test.

Interestingly, this simplified model also performed very well in the other sandwich panel tests at $60^{\circ}$ and $30^{\circ}$ angle of attack, suggesting that it could become the basis of a practical sandwich panel model for destructive re-entry tools.

\subsection{Reaction Wheel Tests}

The first of the reaction wheel tests was planned to provide an indication of the fragmentation of the reaction wheel. As such, the test was run at FC1, with a nominal condition of $100 \mathrm{~kW} / \mathrm{m}^{2}$. The CFD rebuilds suggest that the larger radius of the housing results in a reduced stagnation point heat flux, such that the housing failure would occur later than the $\sim 100$ s failure seen in the aluminium top hat test.

The behaviour of the aluminium housing of the reaction wheel is not exactly the same as observed in the top hat tests. The flow sequence to the failure of the reaction wheel housing is shown in Fig. 18. There is a very bright radiative emission from the pyrolysis of the paint soon after the test start which is shown in the left hand pane. The emission also allows some of the flow structure to be observed. Impingement of the flow on the base/housing connection can be observed, and it is evident that the flow is separated downstream of this location. The heating appears to be mainly on the front surface with the slightly raised housing centre emitting clearly more radiation than the remainder of the test object.

The red emissions indicate the outgassing of zinc, as observed in the top hat tests. There is also evidence of blue outgassing along the sides of the test object. The first evidence of some shape change of the front face occurs at 70 s, with the slightly raised surface appearing to be pushed back slightly. By 90s, the front surface appears flat, and by $97 \mathrm{~s}$, the location of the end of the ball bearing unit (BBU) can be seen as shown in the centre pane of Fig. 18, with the front face of the housing being clearly molten material within an oxide bag. Very minor wobbling of the housing can be observed at $107 \mathrm{~s}$, with the major motion beginning at $122 \mathrm{~s}$. At $125 \mathrm{~s}$, the front face collapses and the housing is blown off, as shown in the right hand pane, and the sides of the housing still appear to be solid whilst the front face is completely removed.

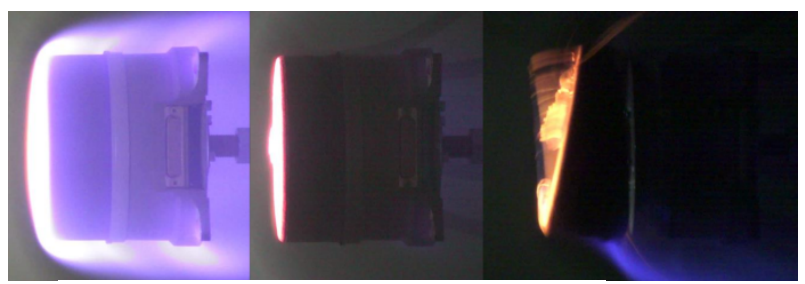

Fig. 18. Reaction Wheel Cover Failure.

The housing was retrieved from the floor of the wind tunnel, and essentially confirms that it was removed in a single piece The lack of damage from landing on the floor of the tunnel suggests that it was still essentially solid at the time it was blown off. The housing removal is possible due to the failure of the glue between the housing and the base.

Once the steel parts are exposed the shock standoff distance is clearly reduced due to the smaller radius of curvature on the BBU. This is consistent with the CFD solutions and suggests that the heat fluxes will be higher on the BBU than they were on the housing. There is no apparent damage to the BBU, flywheel and base in the remainder of the test, but it is clear that some of the internal components are being affected by the temperatures being reached from the outgassing observed. As it became clear that essentially a steady state had been reached, and the base was not going to 
fail at this heat flux, the test was stopped after 30 minutes.

Given the survival of the majority of the reaction wheel structure at the FC1 flow condition, the wind tunnel was set to $\mathrm{FC} 2$, with a nominal heat flux of $200 \mathrm{~kW} / \mathrm{m}^{2}$. The test object had cooled for some time before the re-test, but it had not been dismounted and was still at an elevated temperature of a few hundred degrees Celsius at the start of the test.

Immediately on re-entering the flow, a red outgas could be seen. Again, the heating on the BBU and the edge of the flywheel could be seen from the radiative emission from the surfaces. A clear temperature profile could be seen in the BBU and flywheel, with a cooler region in the centre of the flywheel, which is consistent with CFD results on this configuration. Eventually, the aluminium rear structure became sufficiently molten that the BBU/flywheel structure was able to tear itself from the base under gravity as shown in the centre and right hand panes of Fig. 19. Once the flywheel had been removed, the exposed aluminium of the remaining housing was melted rapidly.

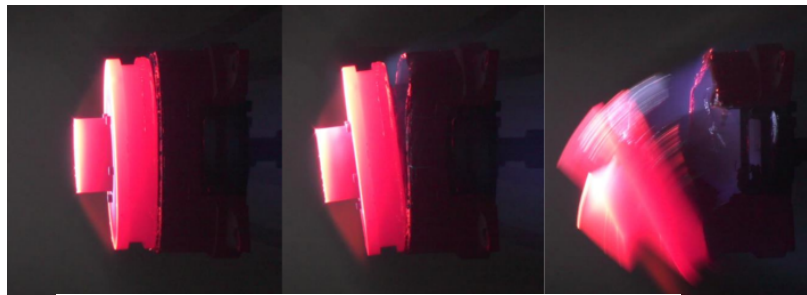

Fig. 19. Reaction Wheel Steel Part Removal.

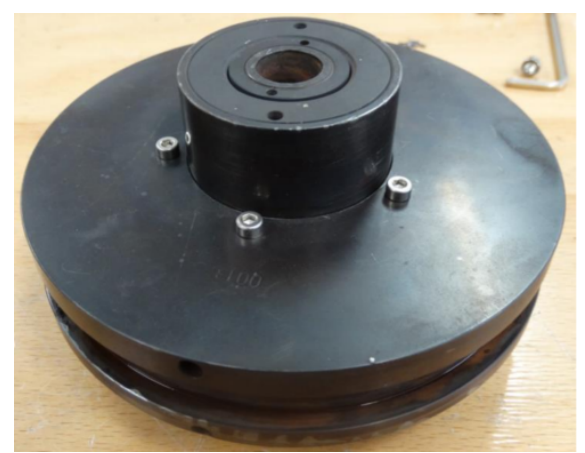

Fig. 20. BBU/Flywheel Test Object.

The intention of the final reaction wheel test at high heat flux was to melt the steel components, and to understand the demisability of these highly survivable parts. To this end, condition FC3 was used, providing heat fluxes of over $1 \mathrm{MW} / \mathrm{m}^{2}$ to the ball bearing unit (BBU) and more than $500 \mathrm{~kW} / \mathrm{m}^{2}$ to the flywheel, based on CFD calculations. It is worth noting at this stage that FC3 is close to the maximum power of the L2K facility.

This test took place a significant time after the first test, and the test object was dismantled and rebuilt. New steel screws were used to join the BBU and flywheel, and the parts were also thermocoupled to provide extra data on the heat transfer. The test object is shown in Fig. 20.

On insertion into the flowfield, green outgas is evident from the flywheel at $4 \mathrm{~s}$, and red outgas from $8 \mathrm{~s}$. At 20s, there is some evidence of surface change on the BBU front face, and it becomes white hot by $40 \mathrm{~s}$. At this stage, the relatively low thermal conductivity of the stainless steel, about $15 \mathrm{~W} / \mathrm{mK}$, maintains a high temperature gradient through the material, and these temperature gradients can be clearly seen in the radiative emission. The front face of the BBU becomes steadily hotter to the point that the radiation is sufficiently strong that the centre hole cannot be seen. At this point there is some emission of very small particles from the BBU, the damage being observable in Fig. 21.

As the heat penetrates further into the BBU shaft, the particle emission stops. This is thought to be due to the reduction of the front face temperature due to the increased heat dispersion through the test object. The increased penetration of heat into the BBU is evident from the apparent sidewall colour. The temperature gradient on the flywheel is also evident with the central separated flow part being relatively cold, and the corner of the flywheel being yellow as shown in Fig. 19. It is noticeable that there is a clear conduction gap between the BBU and the flywheel and a large temperature difference between them is maintained. At approximately $450 \mathrm{~s}$, the object appeared to be in a thermally steady state.

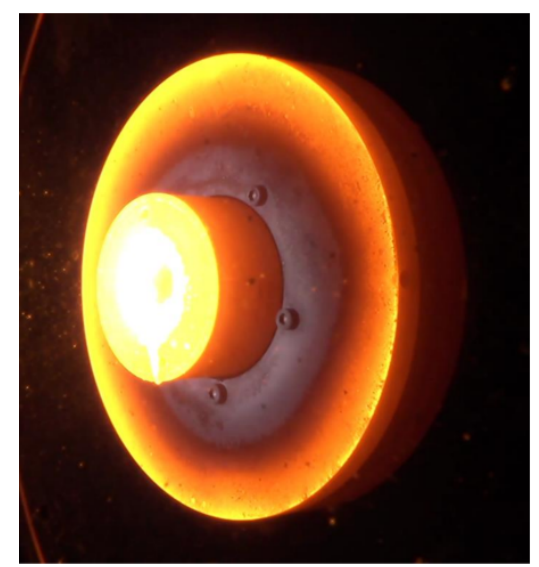

Fig. 21. BBU/Flywheel Under Test.

The non-demise of the test object is driven by the high melting point of the stainless steel components, allied to the large radiating area. As only the front surface is heated, there is a much larger area for radiative cooling. This is confirmed using the thermocouple data and an energy balance. 
Table 4. Reaction Wheel FC3 Energy Balance.

\begin{tabular}{lcc}
\hline & BBU & Flywheel \\
\hline Heat Flux Input $\left(\mathrm{kW} / \mathrm{m}^{2}\right)$ & 1250 & 520 \\
Catalycity & 0.9 & 0.9 \\
Emissivity & 0.87 & 0.87 \\
Test Enthalpy $(\mathrm{MJ} / \mathrm{kg})$ & 13.3 & 13.3 \\
Air enthalpy $(\mathrm{MJ} / \mathrm{kg})$ & 1.46 & 1.12 \\
Front diameter $(\mathrm{m})$ & 0.047 & 0.12 \\
Front hole diameter $(\mathrm{m})$ & 0.014 & 0.047 \\
Visible side length $(\mathrm{m})$ & 0.03 & 0.03 \\
Radiating Area $\left(\mathrm{m}^{2}\right)$ & 0.0060 & 0.0305 \\
Eqbm Temperature $\left({ }^{\circ} \mathrm{C}\right)$ & 1247 & 1012 \\
\hline
\end{tabular}

Predicting an equilibrium temperature for each component through an understanding of the individual heat input and rejection is complex. It is assumed that there is no conduction between the components and that an average temperature value is representative of each part. This latter assumption is not perfect as there are clear temperature gradients, especially in the flywheel, but it is good enough to provide a representative value for the temperatures seen in order to confirm an understanding of the heat transfer through the component. The heat balance shown in Table 4 gives a BBU temperature of $1250^{\circ} \mathrm{C}$ and a flywheel temperature of $1010^{\circ} \mathrm{C}$ which are in very good agreement with the measured thermocouple data.

\subsection{CubeSat Tests}

The demise tests of the CubeSat structure provide some insight into the demise of complex objects, and also form the first test where the GRFP electronics cards are a major focus of a demisability test. As the test is complex, the main interest is phenomenological, and it is expected that quantitative understanding of the test will be limited.

The complete CubeSat is inserted into the flow as shown in the top left hand pane of Fig.22. Within 20s the polyamide plastic structures have failed leaving burning residue on the aluminium frame.

The next stage of the demise of the CubeSat begins with the outgassing of the sound card components. Following this, there is the first failure of the aluminium frame, at the centre of the front structural beam, as seen in the top right pane of Fig. 22. At 35s, there are visible aluminium drops shed from the structure. These drops are of the order of a few millimetres diameter, with their large size again being driven by the oxide film. The corners of the aluminium frame are observed to melt, followed by the top frame material as shown in the bottom right pane of Fig. 22. The sound card outgassing stops at about the same time. The aluminium side struts then begin to melt, and this recession is relatively fast.

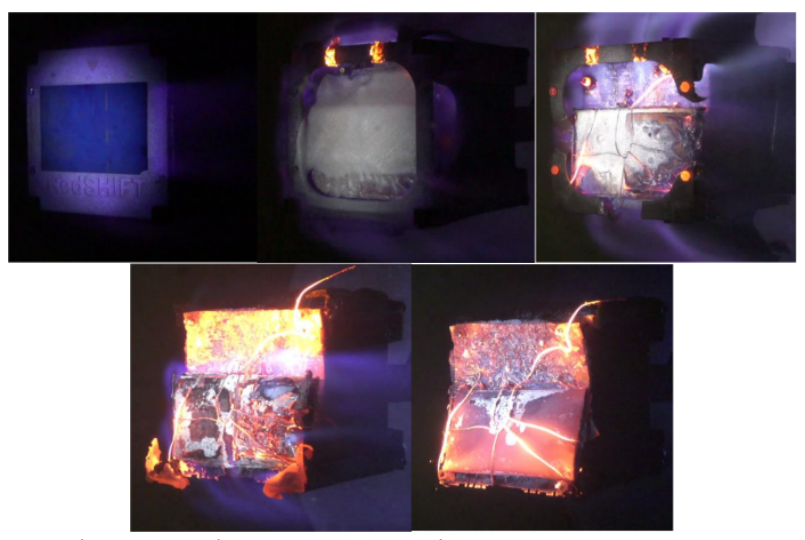

Fig. 22. CubeSat Test Demise.

The battery cover can be seen to have flaked, but not melted, and the GFRP sound card is also hot, but not molten in the bottom right pane of Fig. 22. The aluminium sides are melted down to the card level, and the larger radius of curvature of the overall geometry is evident in the flow structure. At this stage, there is an apparent steady state, although there is a continual slow sag of the structure.

The high speed camera data is very helpful in this case due to the complexity of the phenomena being observed, particularly in the failure behaviour of the aluminium structure. Prior to the melt of the aluminium struts, some warp is evident leading to a dislocation in the strut where the sides are displaced. This is more likely to be due to thermal stresses than aerodynamic forces and is most likely evidence that the material is very close to melt. The behaviour of the oxide film is also very clear.

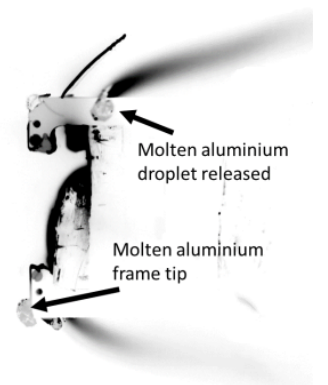

Fig. 23. High Speed Camera Still of Drop Release.

The still shown in Fig. 23 demonstrates a molten part of the structure which is still attached and a large droplet which has been removed from the structure. The molten parts can be seen to collapse and the oxide bag containing the molten material is buffeted under the aerodynamic load, eventually leading to the removal of the droplet when there is sufficient force to tear the oxide layer. For most of the parts, the motion is quite large, suggesting that the material is fully molten inside the oxide layer. This is different behaviour to that seen 
in the top hat tests. The aluminium droplet removal all follows the same process of melt, agglomeration inside an oxide bag, and then shear.

This test shows that the demise of a CubeSat is more complex than had been initially envisaged, and that the electronics cards are less demisable than expected. Given this, it was decided to increase the heat flux and to run condition FC2. This was done without dismounting the CubeSat from the wind tunnel. The CubeSat appeared to survive the shutdown of the wind tunnel with no noticeable effect, which suggests that the structure was still essentially sound. The expectation was that the $200 \mathrm{~kW} / \mathrm{m}^{2}$ heat flux would be sufficient to see the demise of the electronics cards.

On insertion into the flow, the battery outer layers, which had been expected to demise in the first test, quickly fail. The first GFRP melt on the sound card is observed before the battery inner layers fail resulting in some of the battery structure being pressed against the sound card protecting the lower parts. The left hand pane of Fig. 24 is just before this occurs and shows that the GFRP card is charred, but intact.

The right hand pane of Fig. 24 shows the residue of the battery against the sound card, and the melting glass at the top of the sound card. The flow of the glass droplets can be seen, and is very slow. Tendrils of glass form, and the card warps, but does not lose its basic structure. Again, the structure as a whole slumps slightly, and the electronic components are all destroyed, but the GFRP cards are still structurally intact, and the steel rods and spacers which hold the cards in place provide the overall integrity of the structure.

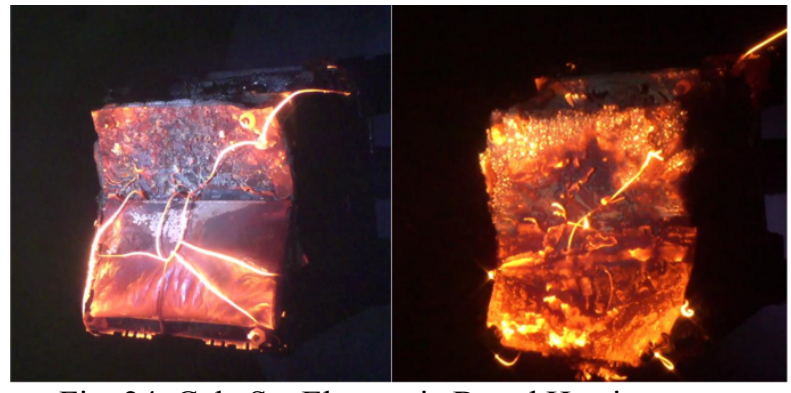

Fig. 24. CubeSat Electronic Board Heating.

As observed in the reaction wheel tests, a large amount of debris was collected from the floor of the wind tunnel post test. This included some aluminium structure parts, electronics components, and molten plastic. A post-test inspection of the remaining CubeSat structure was also undertaken, and a number of images are shown in Fig. 25.

The top panes show the glass which has melted and run across the surface, but also demonstrates that this cannot be considered demised. Higher heat fluxes and/or shear forces would be required to remove the glass from the electronics cards. As these are prevalent on spacecraft, this test indicates that significantly more understanding of the demise of electronics cards is necessary.

The centre panes show the threaded steel rod and spacers which clearly are not demisable at the temperatures reached in these tests. It can be seen that they are indeed the source of the remaining structural integrity of the CubeSat. The shells of the electronics components can also be seen. It is worth noting that the remains are all relatively small, so the risk on the ground would be very low. The issue is whether the steel rods will break, or if the GFRP cards can be removed from them.

The bottom panes show the delamination of the electronics boards to reveal copper layers, and also the remaining rear aluminium frame. The delamination of the boards suggests that in a complete re-entry, where a CubeSat would be expected to reach higher fluxes, the demise of such an object is still likely. However, this test suggests strongly, that some care is required to establish the demisability of even relatively small objects, and that the demise characteristics of common spacecraft components is not well known.

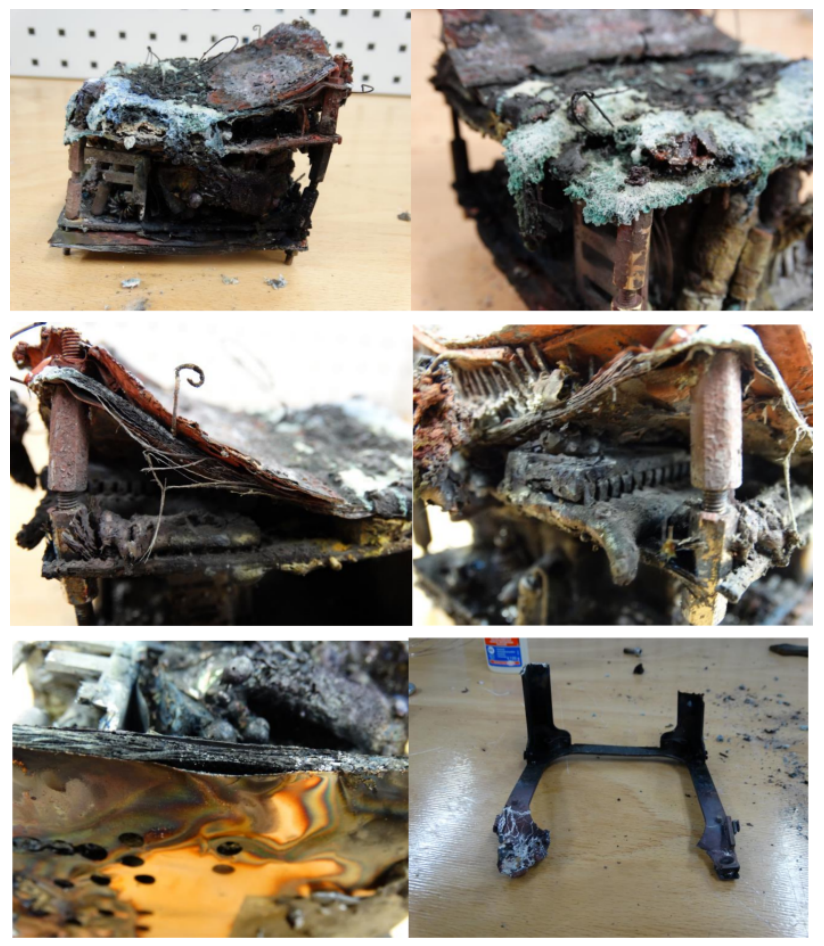

Fig. 25. CubeSat Remains.

\section{Conclusions}

These ground-breaking tests have provided new and useful information on the demise of spacecraft materials and structures. The demisability campaign has covered the behaviour of thin aluminium structures, CFRP facesheets, sandwich panels, and, for the first time 
worldwide, full spacecraft structures. The tests have also been rebuilt using a variety of simplified methods, consistent in complexity with those used in destructive re-entry simulations, to provide additional understanding of the results.

The aluminium tests have shown that the material demise criterion is the most difficult aspect to assess for this material. The strong oxide coating of the material has a much higher melting temperature than the metal itself, and in each case the failure of the material has been seen on the shear of the oxide bag releasing the molten metal. The top hat tests showed catastrophic failure prior to the complete melt of the aluminium, whereas the reaction wheel housing and the CubeSat structure appear to fail after the material in the oxide bag is fully molten.

The CFRP material is seen to be very robust to aerothermodynamic fluxes due to the carbon fibres. The material maintains its integrity for a long time, although some removal of material strips is seen in shear conditions and when the panels are bent. Where a panel experiences significant bending, or shape change, there is much greater damage, and this could potentially be exploited in future design-for-demise activities. It is expected from these results that the material from released CFRP facesheets would survive re-entry.

The behaviour of the panels shows a high sensitivity to the thickness of the CFRP facesheets and a consistent heat transfer behaviour at a range of different angles of attack. The removal of the facesheet is observed on occasion, but it has not been consistent in all tests. Importantly, the flow into the panel, between the facesheets, has provided a honeycomb recession profile which is consistent with the heat flux profile.

The ground breaking tests on the reaction wheel and the CubeSat provide the first data on realistic spacecraft structures. One of the key findings is the sheer number of parts which are released from these structures, especially small electronic components. The reaction wheel tests show a similar failure of the housing as has been observed in the top hat tests, and that the stainless steel parts are extremely resistant to demise. The CubeSat tests demonstrate that the aluminium structures are highly demisable, but that GFRP electronics cards are less demisable than had been assumed. It is also noteworthy that the steel rods used to position the cards in the structure were able to provide continued integrity throughout the test. Although the CubeSat survived the test much more intact than expected, it is still expected that for such a small object, the fluxes later in entry would be sufficiently high for there to be no casualty risk.

\section{Acknowledgements}

This work is funded through the European Commission Horizon 2020, Framework Programme for Research and Innovation (2014-2020), under the ReDSHIFT project (Grant Agreement 687500).

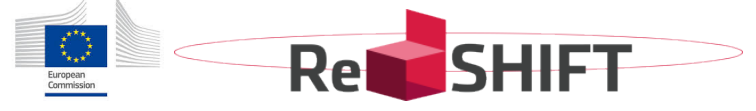

Fluid Gravity Engineering also generously provided their hypersonic chemically reacting CFD code TINA for use in this activity.

\section{References}

[1] J. Carrou, Skylab decay follow-up, Proc. Int. Symp. Spacecraft Flight Dynamics, Darmstadt, 1981.

[2] R. Patera, W. Ailor, The realities of re-entry disposal, $8^{\text {th }}$ AAS/AIAA Space Flight Mechanics Meeting, 1998.

[3] G. Koppenwaller, T. Lips, D. Alwes, Wind tunnel aeroheating and material destruction tests for improved debris re-entry analysis, $5^{\text {th }}$ European Conference on Space Debris, Darmstadt, 2009.

[4] T. Schleutker, A. Guelhan, T. Lips, E. Kaschnitz, B. Bonvoisin, Experimental investigations on the demisability of space relevant materials, ESA Clean Space Industrial Days, Noordwijk, 2016.

[5] A. Pagan, B. Massuti-Ballester, G. Herdrich, J. Merrifield, J. Beck, V. Liedtke, B. Bonvoisin, Investigation of the surface and boundary layer composition for demising aerospace materials, $7^{\text {th }}$ International Workshop on the Radiation of High Temperature Gases in Atmospheric Entry, Stuttgart, 2016.

[6] J. Beck, I. Holbrough, M. Spel, J. Merrifield, Joint fragmentation; phenomena testing and assessment, ESA Clean Space Industrial Days, Noordwijk, 2016.

[7] M. Fittock et al., Methodology and results of high enthalpy wind tunnel and static demisability tests for existing S/C structural joining technologies, $69^{\text {th }}$ International Astronautical Congress, Bremen, 2018.

[8] B. Esser, A. Guelhan, M. Kuhn, Thermal qualification of transpiration cooling for atmospheric entry, $1^{\text {st }}$ CEAS European Air and Space Conference, Berlin, 2007.

[9] J. Beck, I. Holbrough, J. Merrifield, N. Leveque, Design for demise analysis using the SAM destructive re-entry model, in M. Vasile, E. Minisci, L. Summerer, P. McGinty (Eds.) Stardust Final Conference, Springer, 2018.

[10] J. Beck, I. Holbrough, J. Merrifield, N. Joiner, Progress in hybrid spacecraft/object oriented destructive re-entry modelling using the SAM code, $7^{\text {th }}$ European Conference on Space Debris, Darmstadt, 2017. 